\title{
Activation of Sterol Regulatory Element Binding Factors by Fenofibrate and Gemfibrozil Stimulates Myelination in Zebrafish
}

\author{
Yoshifumi Ashikawa1t, Yuhei Nishimura 1,2,3,4,5t, Shiko Okabe', Shota Sasagawa2, \\ Soichiro Murakami', Mizuki Yuge ${ }^{1}$, Koki Kawaguchi' ${ }^{2}$, Reiko Kawase ${ }^{2}$ and \\ Toshio Tanaka ${ }^{2,3,4,5 *}$
}

\begin{abstract}
${ }^{1}$ Department of Molecular and Cellular Pharmacology, Pharmacogenomics and Pharmacoinformatics, Mie University Graduate School of Medicine, Tsu, Japan, ${ }^{2}$ Department of Systems Pharmacology, Mie University Graduate School of Medicine, Tsu, Japan, ${ }^{3}$ Mie University Medical Zebrafish Research Center, Tsu, Japan, ${ }^{4}$ Department of Omics Medicine, Mie University Industrial Technology Innovation Institute, Tsu, Japan, ${ }^{5}$ Department of Bioinformatics, Mie University Life Science Research Center, Tsu, Japan
\end{abstract}

OPEN ACCESS

Edited by: Giovanni Li Volti, University of Catania, Italy

Reviewed by: Maria Violetta Brundo, University of Catania, Italy Massimo Mattia Santoro, University of Leuven-VIB, Belgium

*Correspondence: Toshio Tanaka tanaka@doc.medic.mie-u.ac.jp

tThese authors have contributed equally to this work.

Specialty section: This article was submitted to Experimental Pharmacology and Drug

Discovery,

a section of the journal Frontiers in Pharmacology

Received: 17 May 2016 Accepted: 28 June 2016

Published: 11 July 2016

Citation: Ashikawa Y, Nishimura Y, Okabe S, Sasagawa S, Murakami S, Yuge M,

Kawaguchi K, Kawase R and Tanaka T (2016) Activation of Sterol Regulatory Element Binding Factors by Fenofibrate and Gemfibrozi Stimulates Myelination in Zebrafish.

Front. Pharmacol. 7:206. doi: 10.3389/fphar.2016.00206
Oligodendrocytes are major myelin-producing cells and play essential roles in the function of a healthy nervous system. However, they are also one of the most vulnerable neural cell types in the central nervous system (CNS), and myelin abnormalities in the CNS are found in a wide variety of neurological disorders, including multiple sclerosis, adrenoleukodystrophy, and schizophrenia. There is an urgent need to identify small molecular weight compounds that can stimulate myelination. In this study, we performed comparative transcriptome analysis to identify pharmacodynamic effects common to miconazole and clobetasol, which have been shown to stimulate myelination by mouse oligodendrocyte progenitor cells (OPCs). Of the genes differentially expressed in both miconazole- and clobetasol-treated mouse OPCs compared with untreated cells, we identified differentially expressed genes (DEGs) common to both drug treatments. Gene ontology analysis revealed that these DEGs are significantly associated with the sterol biosynthetic pathway, and further bioinformatics analysis suggested that sterol regulatory element binding factors (SREBFs) might be key upstream regulators of the DEGs. In silico screening of a public database for chemicals associated with SREBF activation identified fenofibrate, a peroxisome proliferator-activated receptor $\alpha$ (PPAR $\alpha$ ) agonist, as a drug that increases the expression of known SREBF targets, raising the possibility that fenofibrate may also stimulate myelination. To test this, we performed in vivo imaging of zebrafish expressing a fluorescent reporter protein under the control of the myelin basic protein (mbp) promoter. Treatment of zebrafish with fenofibrate significantly increased expression of the fluorescent reporter compared with untreated zebrafish. This increase was attenuated by co-treatment with fatostatin, a specific inhibitor of SREBFs, confirming that the fenofibrate effect was mediated via SREBFs. Furthermore, incubation of zebrafish with another PPAR $\alpha$ agonist, gemfibrozil, also

Abbreviations: DEG, differentially expressed gene; dpf, days-post-fertilization; mbp, myelin basic protein; mEpiSC, murine epiblast stem cell; OPC, oligodendrocyte progenitor cell; PPAR, peroxisome proliferator-activated receptor; SREBF, sterol regulatory element binding factor; TF, transcription factor. 
increased expression of the mbp promoter-driven fluorescent reporter in an SREBFdependent manner. These results suggest that activation of SREBFs by small molecular weight compounds may be a feasible therapeutic approach to stimulate myelination.

Keywords: SREBFs, fenofibrate, gemfibrozil, oligodendrocytes, myelination, comparative transcriptomics, zebrafish, systems pharmacology

\section{INTRODUCTION}

Oligodendrocytes are major myelinating cells of the central nervous system (CNS) and are thus critical to proper neuronal functioning. However, they are also an extremely vulnerable cell type, and CNS myelin abnormalities are found in a variety of neurological disorders (reviewed in Chew and DeBoy, 2015), including white matter pathologies associated with brain injury, endocrine and metabolic abnormalities, and psychiatric and neurodegenerative conditions. The aberrant myelination associated with these heterogeneous pathologies can result from the production of myelin with abnormal structure, the virtual absence of myelin, and damage to existing myelin by insults such as oxidative stress, mechanical injury, and inflammation. Ultimately, the loss of normal myelination leads to defective impulse conduction along the nerve fiber.

Current therapeutic approaches are aimed at reducing demyelination by immune-, excitotoxic-, and oxidative stressmediated injury and at promoting myelination through cell engraftment, direct protection of endogenous oligodendrocytes, and enhancement of OPC activity. However, there are some reports of effective non-immune-based remyelinating therapeutics, including benztropine (Deshmukh et al., 2013), miconazole (Najm et al., 2015), clobetasol (Najm et al., 2015; Porcu et al., 2015), and halcinonide (Porcu et al., 2015). Benztropine stimulates myelination by antagonizing the M1/M3 muscarinic receptor (Deshmukh et al., 2013), whereas miconazole and clobetasol stimulate myelination, at least in part, through mitogen-activated protein kinase and glucocorticoid receptor signaling, respectively (Najm et al., 2015). Clobetasol and halcinonide have also been shown to stimulate myelination through activation of Smoothened in the hedgehog signaling pathway and retinoid X receptor $\gamma(\operatorname{RXR} \gamma)$ (Porcu et al., 2015). This common mechanism of action suggests the existence of convergent pathways through which the drugs may stimulate myelination.

In this study, we performed comparative transcriptome analysis of mouse epiblast stem cell-derived OPCs (mEpiSCOPCs) treated with miconazole and clobetasol to identify common mechanisms underlying myelination. We were able to identify a number of genes regulated in common by miconazole and clobetasol. Bioinformatic analysis of these genes revealed that SREBFs may be involved in myelination induced by miconazole and clobetasol, raising the possibility that other SREBF-activating drugs may also stimulate myelination. In silico screening of a public database revealed that the peroxisome proliferator-activated receptor $\alpha(\operatorname{PPAR} \alpha)$ agonist fenofibrate can activate SREBFs, which prompted us to examine the drug's effect on myelination in vivo. Using in vivo imaging of zebrafish expressing a fluorescent reporter protein driven by the mbp promoter, we confirmed that fenofibrate and a second PPAR $\alpha$ agonist, gemfibrozil, could increase mbp promoter activity in a SREBF-dependent manner. Quantitative PCR (qPCR) analysis demonstrated that the PPAR $\alpha$ agonists increased the expression of two genes downstream of SREBFs and mbp. These results suggest that activation of SREBFs may be a convergent pathway for drugs that stimulate myelination.

\section{MATERIALS AND METHODS}

\section{Ethics Statement}

This study was carried out in strict accordance with Japanese law [The Humane Treatment and Management of Animals (2014), Standards Relating to the Care and Management of Laboratory Animals and Relief of Pain (2013), and the Guidelines for Proper Conduct of Animal Experiments (2006) (Science Council of Japan, 2006; Ministry of the Environment of Japan, 2013, 2014)]. All efforts were made to minimize animal suffering. Mie University Institutional Animal Care and Use Committee guidelines state that no approval is required for experiments using zebrafish.

\section{Compounds}

Fenofibrate and gemfibrozil were obtained from Tokyo Chemical Industry (Tokyo, Japan). Methimazole, propylthiouracil, thyroxine, and fatostatin were obtained from Sigma (St. Louis, MO, USA). Stock solutions of these chemicals were prepared by dissolving in dimethyl sulfoxide (Nacalai Tesque, Kyoto, Japan). 2-Phenoxyethanol was obtained from Wako Chemical (Osaka, Japan).

\section{Comparative Transcriptome Analysis of mEpiSC-OPCs Treated with Miconazole or Clobetasol}

To identify genes related to the mechanisms of miconazole- and clobetasol-induced myelination, we downloaded a transcriptome dataset (GSE63804) from Gene Expression Omnibus (Barrett et al., 2009), which was derived from an analysis of the effects of miconazole and clobetasol on the enhancement of myelination by mEpiSC-OPCs (Najm et al., 2015). The raw data from GSE63804 were processed according to previous reports (Trapnell et al., 2009; Liao et al., 2013, 2014). A count-based differential expression analysis was performed using “TCC” (Sun et al., 2013) to identify DEGs in mEpiSC-OPCs treated with miconazole or clobetasol for 2, 6, or $12 \mathrm{~h}$ compared with untreated mEpiSCOPCs, using a false discovery rate of $20 \%$ as the threshold. 
The murine gene symbols of the DEGs were converted to those of the human orthologs using Life Science Knowledge Bank (World Fusion, Tokyo, Japan). The lists of DEGs are shown in Supplementary Tables S1(1-6). DEGs common to mEpiSCOPCs treated with miconazole and clobetasol are shown in Supplementary Table S2.

\section{Bioinformatic Analysis of Genes Regulated by Both Miconazole and Clobetasol}

To identify the biological pathways enriched in the DEGs regulated by both miconazole and clobetasol in mEpiSC-OPCs, we used ClueGO (Bindea et al., 2009) in Cytoscape (Shannon et al., 2003) with the default settings. The biological pathways significantly enriched in the DEGs are shown in Supplementary Tables S3-1 (6 h) and S3-2 (12 h).

To identify TFs potentially regulating the common DEGs, we used iRegulon (Janky et al., 2014) in Cytoscape (Shannon et al., 2003). iRegulon exploits the fact that genes co-regulated by the same TF contain common TF binding sites, and uses gene sets derived from ENCODE ChIP-seq data (Gerstein et al., 2012; Janky et al., 2014). The predicted TFs with normalized enrichment scores $>5$ are shown in Supplementary Tables S4-1 (6 h) and S4-2 (12 h).

\section{Identification of Chemicals That Increase Expression of Genes Targeted by SREBFs}

To identify chemicals that have been reported to increase the expression of genes regulated by SREBFs, we searched the Comparative Toxicogenomics Database (Davis et al., 2015). The database has been successfully used for in silico prediction of biological pathways associated with metal exposure and developmental disorders, followed by experimental validation of the pathway prediction (Ahir et al., 2013). We searched for compounds that increased the expression of HMGCR and SCD. A list of chemicals identified by the in silico screening are shown in Supplementary Tables S5(1) (SCD) and Supplementary Table S5(2) (HMGCR). A list of FDA-approved drugs common to Supplementary Tables S5 $(1,2)$ is shown in Supplementary Table S5(3).

\section{Zebrafish Strains}

We used an albino zebrafish line (Kelsh et al., 1996) obtained from the Max Planck Institute for Developmental Biology (Tübingen, Germany) to make transgenic Tg (mbp: mCitrine) zebrafish, which express the cyan fluorescent protein mCitrine under the control of the mbp promoter (Jung et al., 2010), allowing us to visualize myelin-producing cells. The promoter and part of the first exon of zebrafish mbp (-1794 to +159 bp from the transcription start site) was synthesized by Invitrogen (Carlsbad, CA, USA). The coding region of mCitrine was amplified by polymerase chain reaction using pCS2 +8 NmCitrine (Addgene, Cambridge, MA, USA) as the template. These DNA fragments were cloned into Tol2 vector (Kawakami, 2007) using the In-fusion HD cloning kit (Takara
Bio, Shiga, Japan) to make a circular plasmid (pT2-mbpmCitrine). The pT2-mbp-mCitrine plasmid and transposase mRNA (Kawakami, 2007) were injected into zebrafish embryos at the 1- to 4-cell stage. Larval zebrafish expressing mCitrine in the spinal cord were selected and maintained. Mature F0 zebrafish were mated with albino zebrafish, and F1 zebrafish expressing mCitrine in the spinal cord were selected and maintained. Mature F1 zebrafish were mated with Tg (eno2: Cerulean) zebrafish, which express Cerulean fluorescent protein specifically in neurons (Bai et al., 2007; Sasagawa et al., 2016a), to create Tg (mbp: mCitrine, eno2: Cerulean) zebrafish. Double Tg zebrafish expressing mCitrine and Cerulean in the spinal cord were selected and subsequently maintained and bred according to previously described methods (Westerfield, 2007; Nishimura et al., 2016). Briefly, zebrafish were raised at $28.5 \pm 0.5^{\circ} \mathrm{C}$ with a 14 -h/10-h light/dark cycle. Embryos were obtained via natural mating and cultured in fish medium (0.07 $\mathrm{mM} \mathrm{KCl}, 2 \mathrm{mM} \mathrm{CaCl}_{2}, 0.5 \mathrm{mM} \mathrm{MgSO}_{4}$, and $0.7 \mathrm{mM}$ $\mathrm{NaHCO}_{3}, \mathrm{pH}$ 7.4) until 5 days post-fertilization (dpf), at which point they were used for in vivo imaging analysis. Zebrafish were maintained on living Paramecium spp. from 5 dpf.

\section{In Vivo Imaging of Tg (mbp: mCitrine, eno2: Cerulean) Zebrafish}

Tg (mbp: mCitrine, eno2: Cerulean) zebrafish were exposed to chemicals from $10 \mathrm{~h}$ post-fertilization to $5 \mathrm{dpf}$ in 12 -well plates (20 larvae/well). At $5 \mathrm{dpf}$, zebrafish were anesthetized with 2-phenoxyethanol and placed in a 96-well imaging plate (ZF plate, Hashimoto Electric Industry, Mie, Japan). In vivo imaging and quantitative analysis of the mCitrine fluorescent signal was performed using ImageXpress Micro with customized programs (Molecular Device, Sunnyvale, CA, USA). Briefly, the image was first processed to identify the region of hindbrain and spinal cord using the eno2 promoter-driven Cerulean fluorescence signal (green borders shown in Figures 4 and 5). The mbp promoterdriven mCitrine fluorescence signals within this region were then measured (red dots shown in Figures 4 and 5). Bright-field images of zebrafish were captured using an SMZ25 microscope (Nikon, Tokyo, Japan).

\section{Quantitative PCR Analysis}

Total RNA was extracted from zebrafish at $5 \mathrm{dpf}$ using a Nucleospin RNA XS kit (Takara, Kyoto, Japan) according to the manufacturer's protocol. cDNAs were generated using a ReverTra Ace qPCR RT Kit (Toyobo). qPCR was performed using an ABI Prism 7300 PCR system (Life Technologies, Carlsbad, CA, USA) with THUNDERBIRD SYBR qPCR Mix (Toyobo). The thermal cycling conditions were: $95^{\circ} \mathrm{C}$ for $1 \mathrm{~min}$, followed by 40 cycles of $95^{\circ} \mathrm{C}$ for $15 \mathrm{~s}, 60^{\circ} \mathrm{C}$ for $15 \mathrm{~s}$, and $72^{\circ} \mathrm{C}$ for $45 \mathrm{~s}$. We measured the expression of 3-hydroxy-3-methylglutarylCoA reductase ( $h m g c r), 7$-dehydrocholesterol reductase ( $\mathrm{h} h \mathrm{cr} 7$ ), mbp, and eukaryotic translation elongation factor 1 alpha 1 (ef1a). hmgcr, dhcr7, and $m b p$ mRNA levels were normalized to efla mRNA levels to correct for variability in the initial template concentration and the conversion efficiency of the 
reverse transcription reaction. The primer sequences are shown in Supplementary Table S6.

\section{Statistical Analysis}

Statistical analysis was performed using Prism 6 (GraphPad, La Jolla, CA, USA). Group means were compared by analysis of variance. Alpha was set at 0.05 . Dunnett's and Tukey's multiple comparisons tests were used for post hoc analysis of the in vivo imaging and qPCR data, respectively, when significant effects were found by analysis of variance. Data are shown as the mean \pm standard error (SEM).

\section{RESULTS}

\section{Identification of Differentially Expressed Genes Regulated by Both Miconazole and Clobetasol}

To identify common mechanisms underlying myelination induced by miconazole and clobetasol, we downloaded a transcriptome dataset from an analysis of the effects of the two drugs on mEpiSC-OPCs (Najm et al., 2015) from a public database (Barrett et al., 2009). Using a false discovery rate of $20 \%$ as the threshold, we identified 79 and 30 DEGs in mEpiSC-OPCs treated for $2 \mathrm{~h}$ with miconazole and clobetasol, respectively [Figure 1, Supplementary Tables S1(1,2)]. Five DEGs were common to both treatments (Supplementary Table S2). We also identified 322 and 65 DEGs in mEpiSC-OPCs treated for $6 \mathrm{~h}$ with miconazole and clobetasol, respectively [Figure 1, Supplementary Tables S1 $(3,4)]$. Twenty DEGs were common to both treatments (Supplementary Table S2). We also identified 90 and 899 DEGs in mEpiSC-OPCs treated for $12 \mathrm{~h}$ with miconazole and clobetasol, respectively [Figure 1, Supplementary Tables S1 $(5,6)]$. Fifty-three DEGs were common to both treatments (Supplementary Table S2). The change in expression of these common DEGs was the same, with the exception of Abcal that was downregulated and upregulate by miconazole and clobetasol, respectively (Supplementary Table S2). Taken together, these data suggest that genes dysregulated in both miconazole- and clobetasol-treated cells may be involved in a common mechanism to promote myelination.

\section{Identification of Cholesterol Biosynthesis as the Key Biological Pathway Enriched in Genes Regulated by Both Miconazole and Clobetasol}

To identify biological processes enriched in the DEGs regulated by both miconazole and clobetasol, we used ClueGO, a bioinformatics tool that has been used successfully to identify biological functions associated with given gene sets (Bindea et al., 2009; Sasagawa et al., 2016b). ClueGO identified 19 and 20 biological pathways significantly enriched in the 20 (6 h) and 53 (12 h) DEGs, respectively, regulated by both miconazole and clobetasol [Figure 2, Supplementary Tables S3(1,2)]. There were no biological pathways significantly enriched in the five DEGs regulated in common by both miconazole and

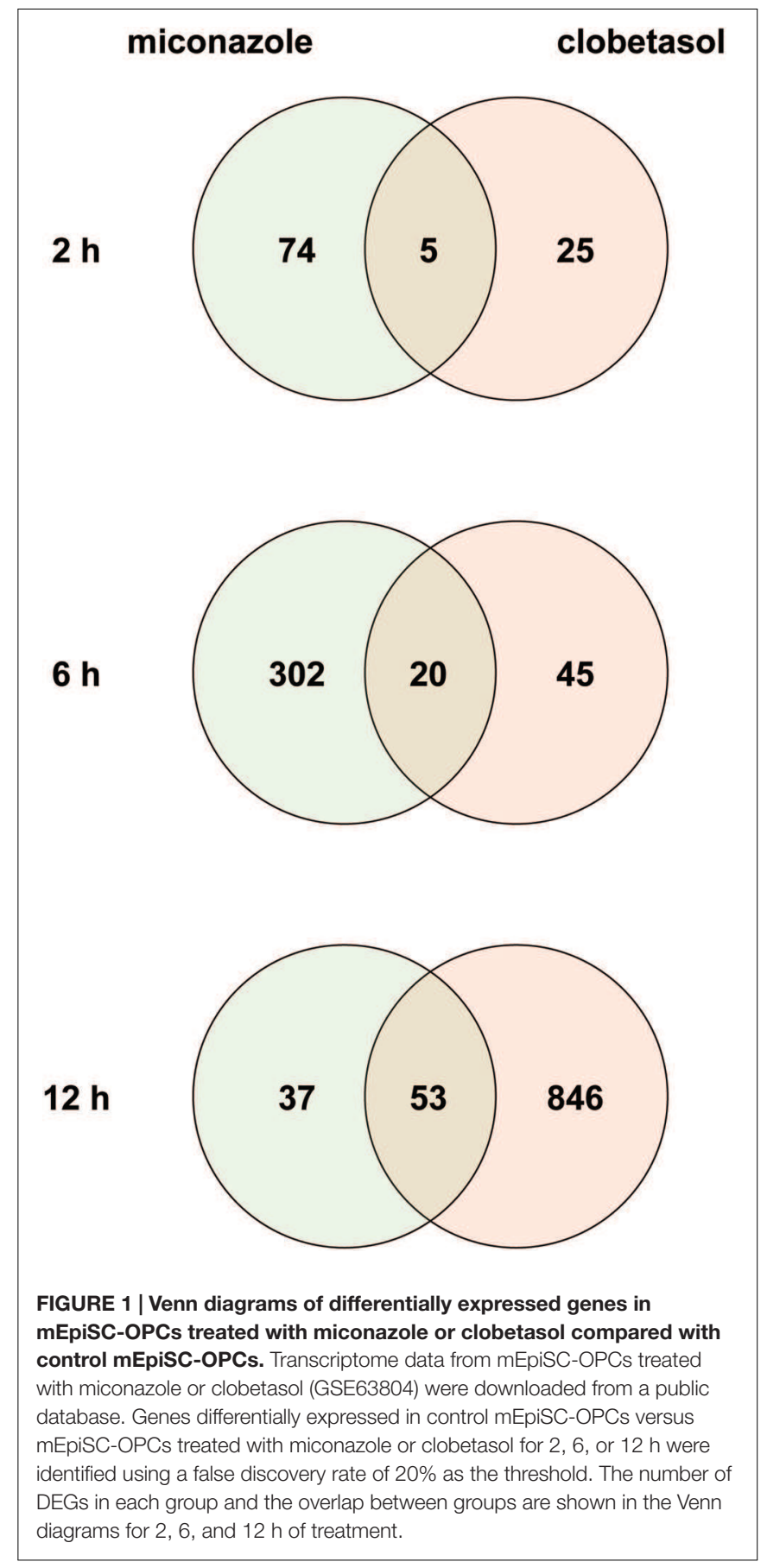

clobetasol at $2 \mathrm{~h}$, possibly because of the small number of DEGs. The 19 biological pathways identified at $6 \mathrm{~h}$ were clustered into three groups; sterol biosynthetic process, fatty acid biosynthetic process, and isoprenoid biosynthetic process [Supplementary Table S3(1)]. The 20 biological pathways at $12 \mathrm{~h}$ were clustered into four groups; sterol biosynthetic process, fatty acid biosynthetic process, "de novo" posttranslational protein folding, and glycolytic process through fructose-6-phosphate [Supplementary Table S3(2)]. These results suggest that sterol biosynthesis and fatty acid biosynthetic processes may be key 


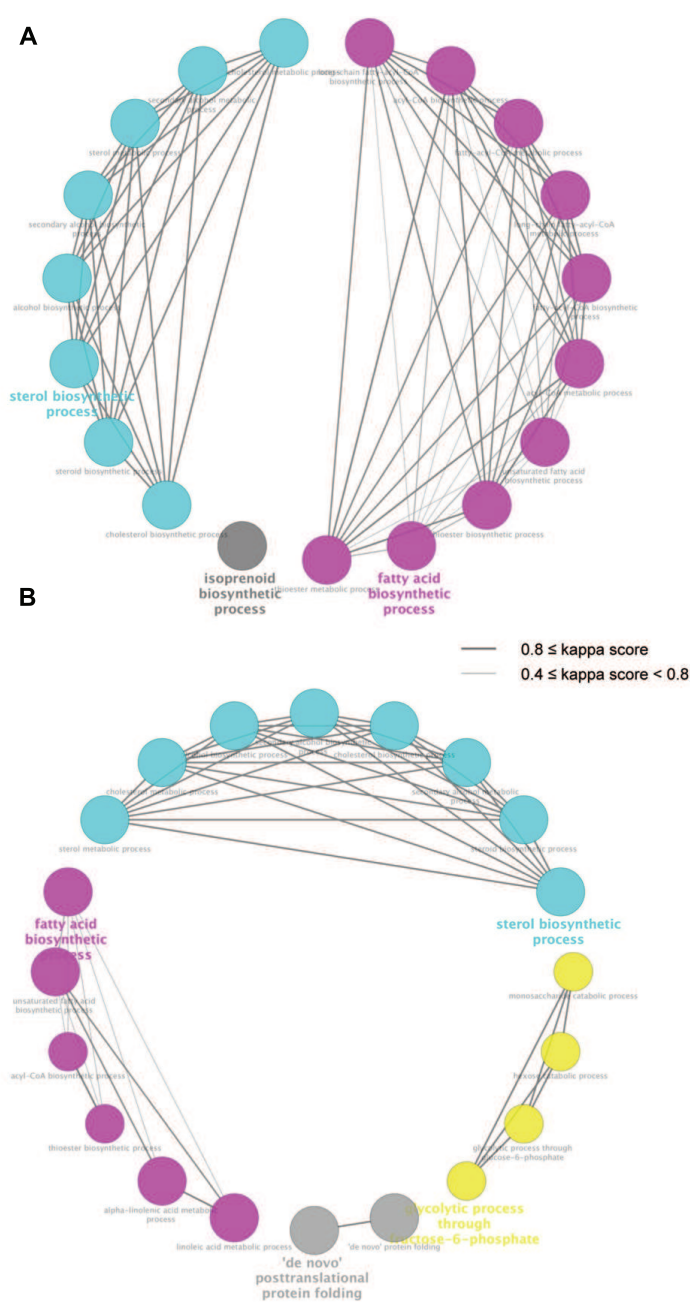

FIGURE 2 | Biological pathways significantly enriched in genes regulated by both miconazole and clobetasol. DEGs common to mEpiSC-OPCs treated with miconazole or clobetasol for 6 or $12 \mathrm{~h}$ were independently subjected to ClueGO using Gene Ontology Biological Pathway as the database. The pathways significantly enriched in the common DEGs at 6 and $12 \mathrm{~h}$ treatment are shown in $(\mathbf{A}, \mathbf{B})$, respectively. Each circle represents one biological pathway. Pairs of biological pathways with similar kappa scores are connected by lines. Biological pathways clustered in the same group are shown in the same color.

pathways involved in miconazole- and clobetasol-stimulated myelination.

\section{Identification of SREBFs as Important Transcription Factors Regulating the Expression of Differentially Expressed Genes Common to Miconazole and Clobetasol}

To identify TFs potentially regulating the DEGs common to mEpiSC-OPCs treated with miconazole or clobetasol, we used iRegulon, which has been used successfully to identify important TFs from given gene sets (Nishimura et al., 2015b; Sasagawa et al., 2016a,b). Based on the list of common DEGs at 6 and $12 \mathrm{~h}$ (Supplementary Table S2), the iRegulon analysis identified SREBF1 and SREBF2 [Figure 3, Supplementary Tables S4 $(1,2)$ ], which is consistent with previous studies showing that SREBF activation increases the expression of 3-hydroxy3-methylglutaryl-CoA reductase (HMGCR) (Vallett et al., 1996; Bennett et al., 2004), stearoyl-CoA desaturase (SCD) (Tabor et al., 1999), cytochrome P450 family 51 subfamily A polypeptide 1 (CYP51A1) (Halder et al., 2002), acyl-CoA synthetase shortchain family member 2 (ACSS2) (Ikeda et al., 2001), and 7dehydrocholesterol reductase (DHCR7) (Prabhu et al., 2014). SREBFs are also known to be important regulators of sterol biosynthesis (Ye and DeBose-Boyd, 2011) and myelination (Norrmen et al., 2014). There were no TFs significantly enriched in the five DEGs regulated by both miconazole and clobetasol for $2 \mathrm{~h}$, probably owing to the limited number of DEGs. These results suggest that activation of SREBFs and the subsequent increase in expression of these SREBF target genes may be involved in the pro-myelinating effects of miconazole and clobetasol.

\section{Identification of Fenofibrate as a Drug That Increases the Expression of Genes Regulated by SREBFs}

From our findings with miconazole and clobetasol, we hypothesized that chemicals that increase the expression of the SREBF target genes might stimulate myelination. To test this, we searched the Comparative Toxicogenomics Database (Davis et al., 2015) for chemicals able to upregulate expression of SCD, identified as the common DEG at 2, 6, and $12 \mathrm{~h}$, and of HMGCR, identified as the common DEG at 2 and $6 \mathrm{~h}$ (Figure 2, Supplementary Table S2). The Comparative Toxicogenomics Database is a public database of the relationships between chemicals and various parameters, including gene expression, curated from the scientific literature. This in silico screening identified nine FDA-approved drugs that increase expression of both SCD and HMGCR [Supplementary Table S5(3)]. It is noteworthy that the list includes clozapine and haloperidol, two antipsychotic medications used to treat schizophrenia. Both compounds are known to activate SREBF and upregulate the transcription of SREBF target genes (Ferno et al., 2006). The $\operatorname{PPAR} \alpha$ agonist fenofibrate has also been shown to activate SREBF2 (Rampler et al., 2003) and to have therapeutic potential for the treatment of adrenoleukodystrophy (Berger et al., 2010), consistent with the possibility that this drug may stimulate myelination.

\section{Fenofibrate and Gemfibrozil Increase Expression of a Fluorescent Reporter Protein Regulated by the Myelin Basic Protein Promoter in Zebrafish}

To investigate whether fenofibrate and a second PPAR $\alpha$ agonist, gemfibrozil, could increase myelin expression in vivo, we examined Tg (mbp: mCitrine, eno2: Cerulean) zebrafish, which express two fluorescent reporters: Cerulean in neurons (driven by the eno 2 promoter) and mCitrine in oligodendrocytes (driven by the mbp promoter). $\mathrm{MBP}$ is the major constituent of 


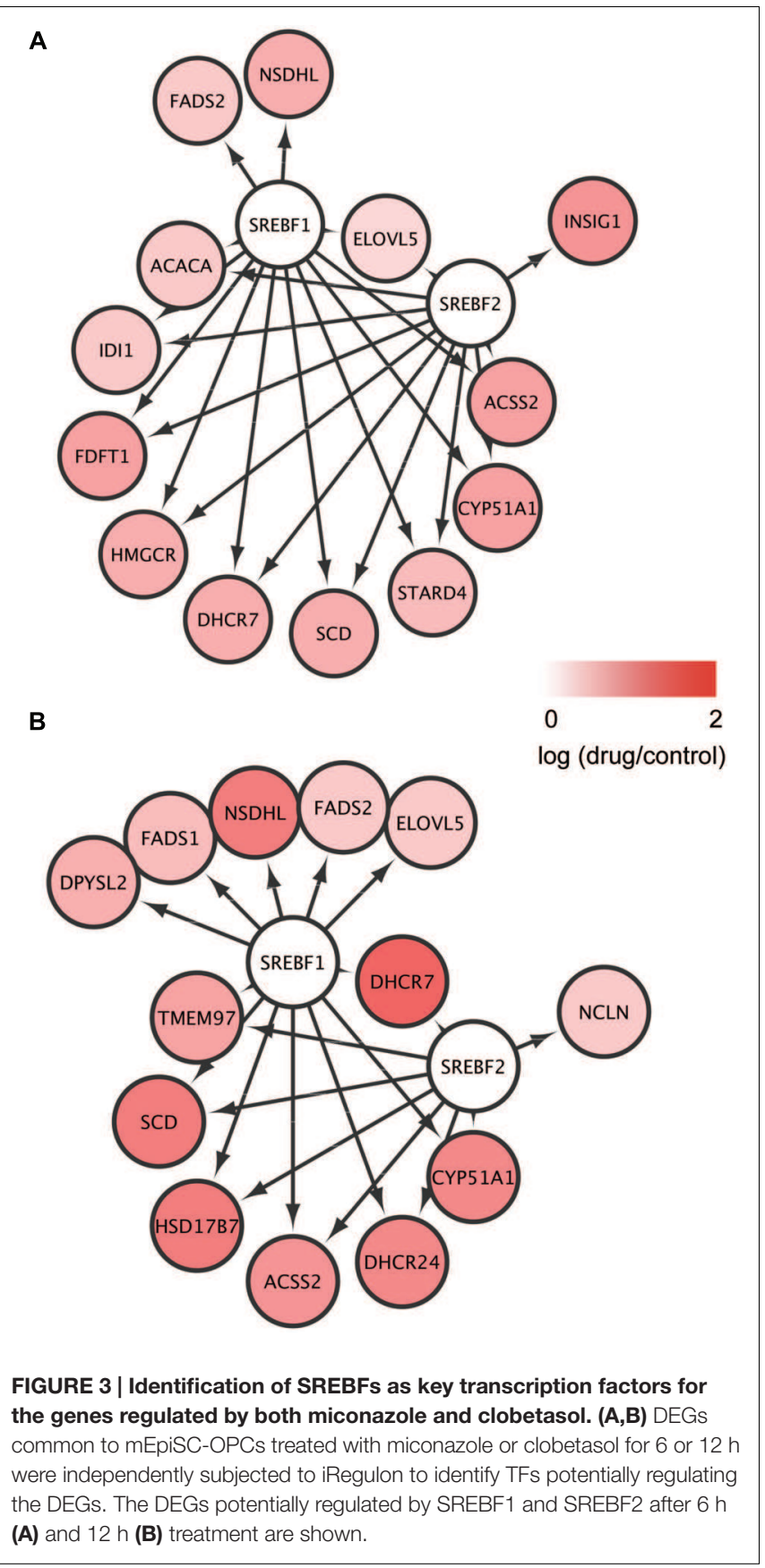

the myelin sheath and is produced by oligodendrocytes. To validate in vivo fluorescent imaging of zebrafish as an assay for examining the effects of chemicals on CNS myelination, we first treated the animals with methimazole (MMI) and propylthiouracil (PTU), which are known to decrease CNS myelination, or with thyroxine, which increases it (Jagannathan et al., 1998; Gilbert et al., 2012). To quantify the effects on myelin promoter activity, we measured the fluorescence intensity of mCitrine within the Cerulean-positive area of the CNS in living zebrafish and found that MMI and PTU dose-dependently decreased the mCitrine signal, whereas thyroxine dose-dependently increased it (Figure 4). These results suggest that in vivo imaging of mbp promoter-driven mCitrine expression in zebrafish can be used to assess the effects of chemicals on myelination.

We then examined the effects of fenofibrate and gemfibrozil on the transgenic zebrafish and found that both fenofibrate and gemfibrozil significantly increased the mCitrine fluorescence (Supplementary Figure S1). To determine the involvement of SREBFs in this increase, we co-treated zebrafish with fenofibrate or gemfibrozil and fatostatin, a specific inhibitor of SREBFs (Kamisuki et al., 2009). Notably, fatostatin reduced the mCitrine fluorescence signal in zebrafish treated with fenofibrate or gemfibrozil to the level seen in control zebrafish (Figure 5). To exclude the possibility that the changes in mCitrine fluorescence result from drug toxicity, we examined the zebrafish by brightfield microscopy. As shown in Supplementary Figure S2, the drug-treated animals showed no malformations, except in the presence of MMI and PTU at $2 \mathrm{mM}$. Thus, it is unlikely that the observed changes in mCitrine fluorescence are due to drug-related toxicity. To examine whether SREBFs may be activated in zebrafish treated with fenofibrate or gemfibrozil, we quantified expression of the SREBF target genes $h m g c r$ and dhcr7. As shown in Supplementary Figure S3, hmgcr mRNA levels were significantly increased in zebrafish treated with either fenofibrate or gemfibrozil. dhor 7 mRNA levels were also increased by both drugs, but the change was significant only in gemfibrozil-treated zebrafish. These results suggest that both fenofibrate and gemfibrozil may activate SREBFs in zebrafish. We also quantified the expression of mbp mRNA to determine its association with the increase in mCitrine fluorescence in fenofibrate- and gemfibrozil-treated zebrafish. As shown in Supplementary Figure S3, mbp mRNA levels were increased by both drugs, with gemfibrozil having a statistically significant effect. These results suggest that the fenofibrateand gemfibrozil-induced increases in mCitrine fluorescence may correlate positively with the increase in mbp mRNA. Taken together, these results suggest that fenofibrate and gemfibrozil may stimulate myelination through activation of SREBFs in zebrafish.

\section{DISCUSSION}

In this study, we demonstrated that activation of SREBFs might be involved in myelination induced by miconazole and clobetasol. We also demonstrated that fenofibrate and gemfibrozil increase mbp promoter-driven expression of a fluorescent reporter protein in zebrafish in a SREBF-dependent manner, suggesting that the pro-myelinating effects of fenofibrate and gemfibrozil occur through SREBF activation.

\section{Myelination Is Induced by Activation of SREBFs}

Using comparative transcriptome analysis, we demonstrated that SREBF activation may be a common mechanism underlying myelination stimulated by miconazole and clobetasol. These results are consistent with previous reports supporting a role 
A

control

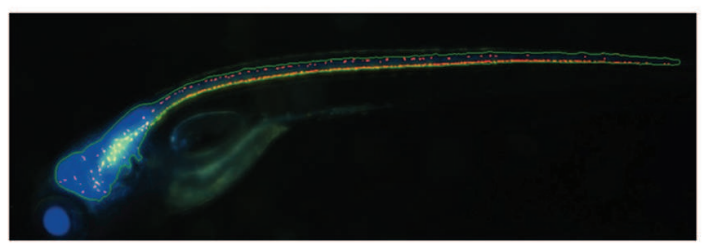

methimzaole (MMI) $2 \mathrm{mM}$

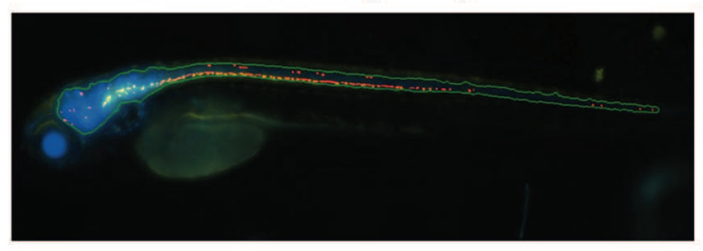

propylthiouracil (PTU) $2 \mathrm{mM}$

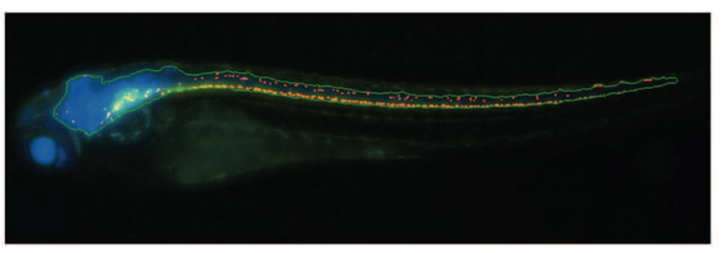

thyroxine (T4) $30 \mathrm{nM}$

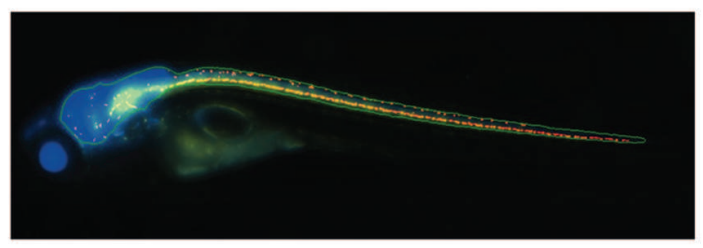

B
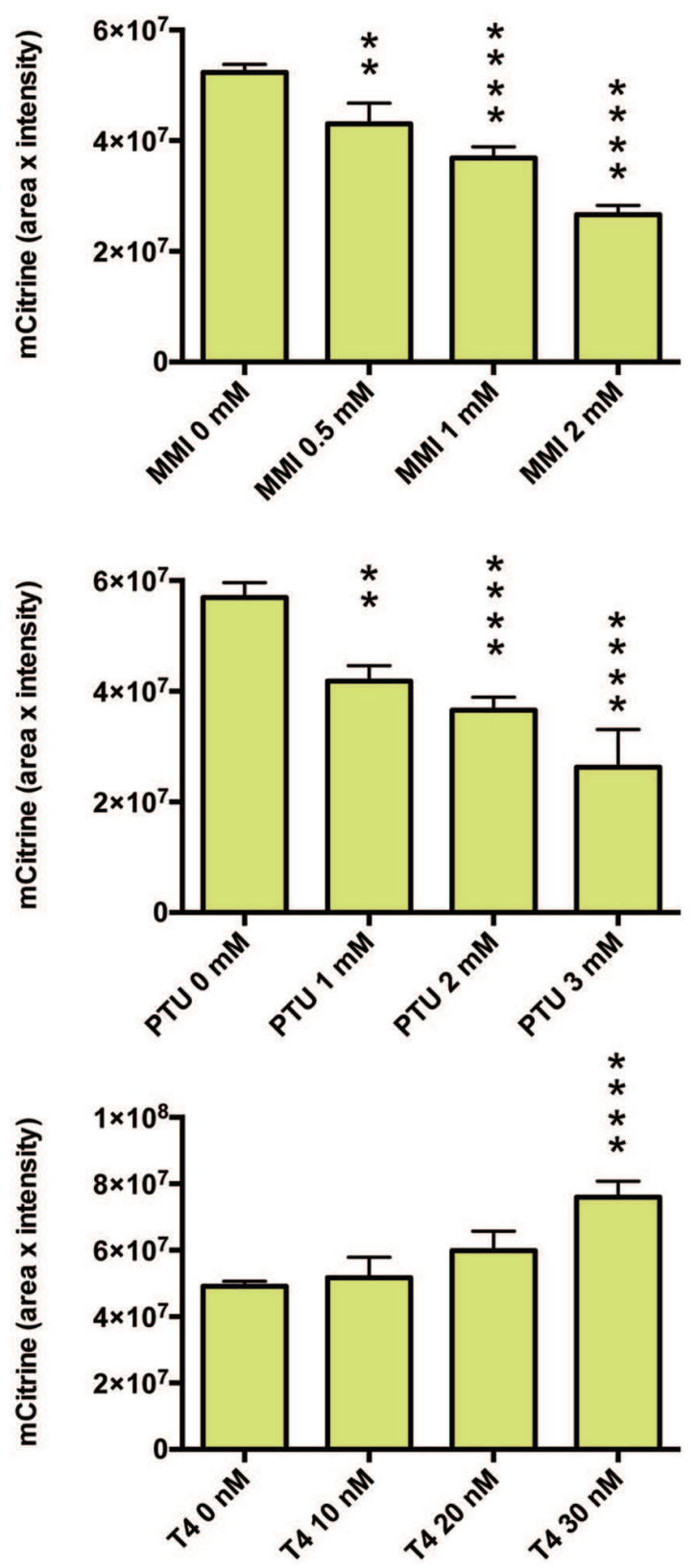

FIGURE 4 | Effects of thyroid hormone modulators on mbp promoter-driven fluorescence in zebrafish. (A) Representative images from in vivo analysis of $\mathrm{Tg}$ (mbp: mCitrine, eno2: Cerulean) zebrafish incubated with or without the indicated concentrations of methimazole (MMI), propylthiouracil (PTU), or thyroxine (T4). (B) Quantification of mCitrine fluorescence intensity within the area of Cerulean fluorescence. (Top) Zebrafish were untreated $(n=40)$ or treated with MMI $(n=16$ for $0.5 \mathrm{mM}, n=26$ for $1 \mathrm{mM}, n=27$ for $2 \mathrm{mM}$ ). (Middle) Zebrafish were untreated $(n=11)$ or treated with PTU ( $n=5$ for $1 \mathrm{mM}, n=10$ for $2 \mathrm{mM}, n=3$ for $3 \mathrm{mM}$ ). (Bottom) Zebrafish were untreated $(n=20)$ or treated with T4 ( $n=6$ for $10 \mathrm{nM}, n=5$ for $20 \mathrm{nM}, n=9$ for $30 \mathrm{nM})$. ** $p<0.01, * * * p<0.0001$ compared with control. 
A

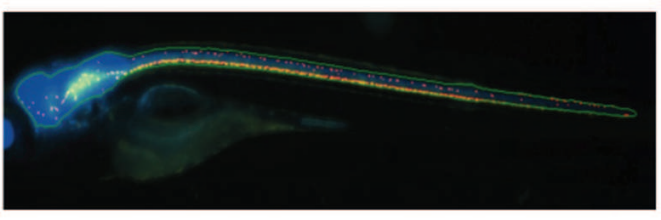

fenofibrate $700 \mathrm{nM}$

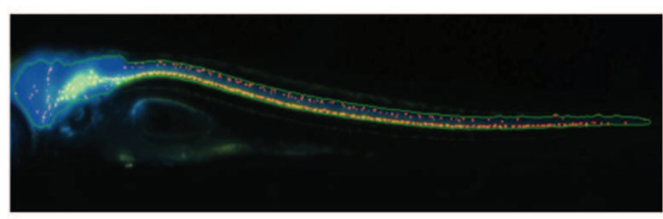

gemfibrozil $750 \mathrm{nM}$

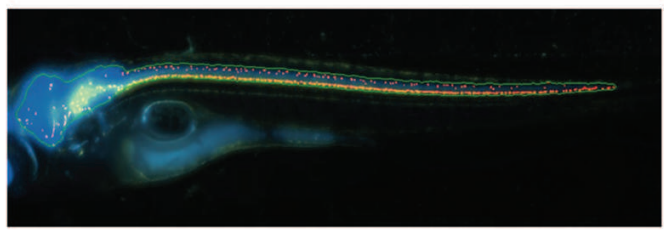

B

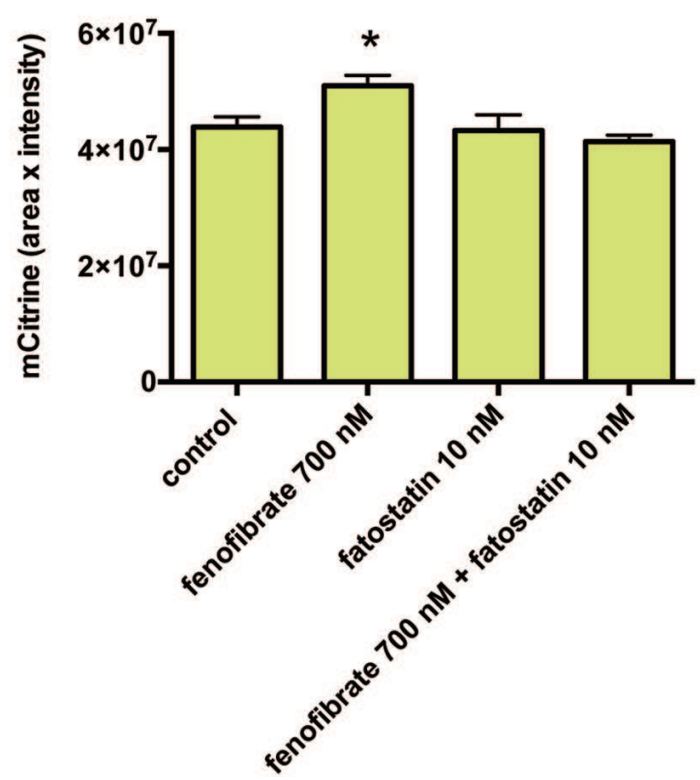

fatostatin $10 \mathrm{nM}$

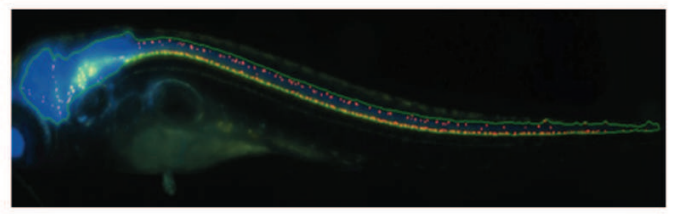

fatostatin $10 \mathrm{nM}+$ fenofibrate $700 \mathrm{nM}$

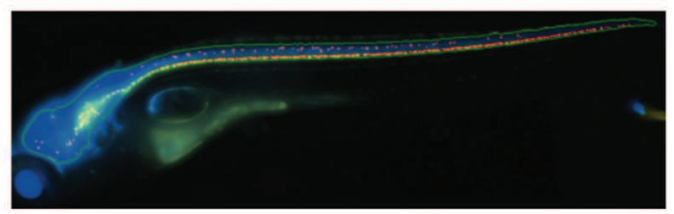

fatostatin $10 \mathrm{nM}+$ gemfibrozil $750 \mathrm{nM}$

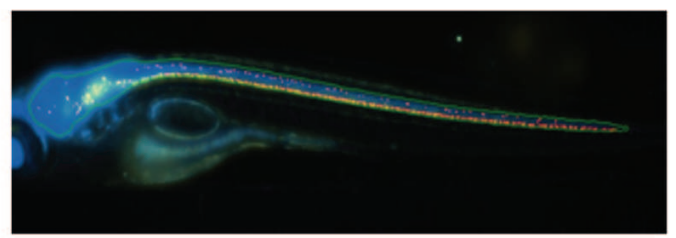

C

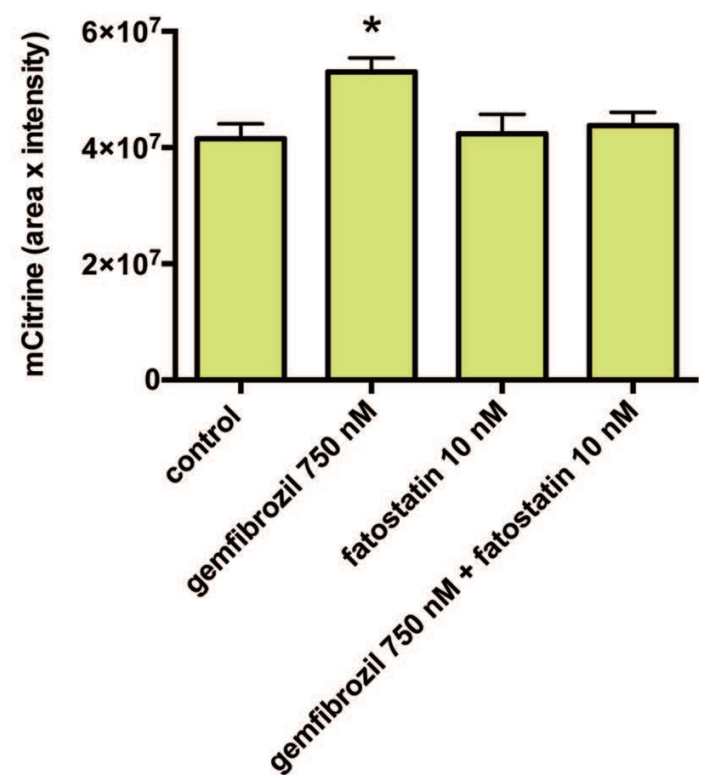

FIGURE 5 | Fenofibrate and gemfibrozil increase mbp promoter-driven fluorescent reporter expression through activation of SREBFs.

(A) Representative images from in vivo analysis of Tg (mbp: mCitrine, eno2: Cerulean) zebrafish incubated with or without the indicated concentrations of fenofibrate, gemfibrozil, and fatostatin. (B,C) Quantification of mCitrine fluorescence intensity within the area of Cerulean fluorescence. (B) Zebrafish were untreated $(n=28)$ or treated with fenofibrate $(n=27)$, fatostatin $(n=20)$, or fenofibrate and fatostatin $(n=5) .{ }^{*} p<0.05$ compared with control. (C) Zebrafish were untreated ( $\left.n=7\right)$ or treated with gemfibrozil $(n=7)$, fatostatin $(n=8)$, or gemfibrozil and fatostatin $(n=8)$. * $p<0.05$ compared with control. 
for SREBFs in myelination. For example, mammalian target of rapamycin complex 1 (mTORC1) regulates oligodendrocyte differentiation and myelination through activation of SREBFs (Norrmen et al., 2014), and mice carrying mutations in SREBF cleavage activating protein (SCAP), a SREBF activator, show congenital hypomyelination (Verheijen et al., 2009). Although SREBFs have not previously been associated with the pharmacological effects of miconazole and clobetasol, the transcriptome analysis revealed cholesterol biosynthesis as a pathway significantly enriched in genes perturbed by both drugs (Najm et al., 2015). Collectively, our data and these observations strongly suggest that the pro-myelinating effects of miconazole and clobetasol are dependent on SREBF activation.

We also demonstrated that known SREBF target genes, including HMGCR (Vallett et al., 1996; Bennett et al., 2004), SCD (Tabor et al., 1999), CYP51A1 (Halder et al., 2002), ACSS2 (Ikeda et al., 2001), and DHCR7 (Prabhu et al., 2014), are differentially upregulated in mEpiSC-OPCs treated with both miconazole and clobetasol. Expression of SCD positively correlates with peripheral axonal myelination (Garbay et al., 1998). Increased HMGCR expression is the predominant mechanism by which myelination is induced by neuregulin 1 in Schwann cells (Pertusa et al., 2007). These findings suggest that increased expression of SCD and HMGCR may stimulate myelination.

Using in silico screening of the Comparative Toxicogenomics Database (Davis et al., 2015), we identified nine FDA-approved drugs that increase the expression of both SCD and HMGCR.

Clozapine and haloperidol are known to increase $S C D$ and HMGCR expression through activation of SREBFs (Ferno et al., 2006), and clozapine also promotes myelin lipid synthesis in cultured oligodendrocytes (Steiner et al., 2014). Progesterone stimulates remyelination in mouse models of demyelinating diseases (Chew and DeBoy, 2015). Amiodarone increases phospholipid levels, possibly through induction of genes associated with cholesterol synthesis (Antherieu et al., 2011). Although we are unaware of previous reports of a relationship between the PPAR $\gamma$ agonist troglitazone and myelination, one study showed that another PPAR $\gamma$ agonist, pioglitazone, does stimulate myelination (Eto et al., 2008). Thus, chemicals that can activate SREBFs and increase the expression of SCD and HMGCR may stimulate myelination.

\section{Fenofibrate and Gemfibrozil Stimulate Myelination by Activating SREBFs}

We used zebrafish to examine the effects of fenofibrate and gemfibrozil on myelination in vivo. In a survey of 1318 human drug targets, $86 \%$ had orthologs in zebrafish (Gunnarsson et al., 2008). This high conservation makes the zebrafish a useful animal model for drug screening. Although there can be interspecies differences in drug pharmacodynamics, several studies have shown that zebrafish can be used successfully to identify novel drugs for treatment of human diseases and to examine the safety and toxicity of drugs in preclinical studies (Barros et al., 2008; Helenius and Yeh, 2012; Mathias et al., 2012; Rihel and Schier, 2012; Esterberg et al., 2013). Zebrafish have also been used as a model organism to investigate CNS myelination (Mathews et al., 2014; Preston and Macklin, 2015). The structure of myelin and the molecular mechanism underlying myelination are well conserved between zebrafish and mammals (Preston and Macklin, 2015). The ease with which zebrafish can be genetically manipulated, their transparency, and their ability to absorb a wide range of chemicals from the surrounding medium make zebrafish a highly suitable model for in vivo chemical screening (Nishimura et al., 2015a; Rennekamp and Peterson, 2015). Indeed, zebrafish have been used successfully to identify novel compounds with pro-myelinating properties (Buckley et al., 2010). Using in vivo imaging of zebrafish, we were able to demonstrate that fenofibrate and gemfibrozil increased the activity of the mbp promoter via activation of SREBFs.

Several mechanisms can be proposed by which fenofibrate and gemfibrozil might activate SREBFs. Both compounds are PPAR $\alpha$ agonists (Friedland et al., 2012), and activation of PPAR $\alpha$ can stimulate SREBF signaling through multiple mechanisms, including increasing SREBF expression (Rampler et al., 2003; Martens et al., 2008; Yan et al., 2014), enhancing SREBF proteolytic cleavage (Knight et al., 2005), and increasing SREBF activity via recruitment of transcriptional co-activators (van der Meer et al., 2010). Gemfibrozil activates PPAR $\beta / \delta$ (Ogata et al., 2009; Roy et al., 2013) and increases the expression of myelin in human oligodendrocytes through $\operatorname{PPAR} \beta / \delta$ activation (Jana et al., 2012). PPAR $\beta / \delta$ activation can also stimulate SREBF signaling (Yang et al., 2014). Fibrates have been considered as potential therapeutics for diseases associated with impaired oligodendrocytes, such as multiple sclerosis, adrenoleukodystrophy, schizophrenia, and traumatic brain injury (Besson et al., 2005; Yang et al., 2008; Berger et al., 2010; Rolland et al., 2012; MandrekarColucci et al., 2013). Further studies are required to determine whether fenofibrate and gemfibrozil can stimulate myelination through activation of SREBFs in these neurological disorders.

\section{AUTHOR CONTRIBUTIONS}

YN conceived the study, performed the bioinformatics analysis, generated the transgenic zebrafish, and wrote the paper. YA performed experiments to validate the effects of chemicals on myelination in zebrafish. SO analyzed the transcriptome data. RK generated the transgenic zebrafish. SS, SM, MY, SO, and KK provided assistance with experiments. TT conceived the study and wrote the paper.

\section{ACKNOWLEDGMENTS}

This work was supported, in part, by the Japan Society for the Promotion of Science KAKENHI (25670127, 15K15051, 24510069), JST A-step (AS262Z00004Q), and Long-range Research Initiative of the Japan Chemical Industrial Association (13_PT01-01). We acknowledge Edward Burton for sharing the 
sequence of the zebrafish eno2 promoter. We are grateful to Mahomi Suzuki, Mitsunari Yamaguchi, and Yasutomo Kubota (Molecular Device) for creating the excellent programs used in ImageXpress Micro. We also thank Junko Koiwa, Yuka Hayakawa, Hiroko Nakayama, Yuka Takahashi, Chizuru Hirota, and Michiko Ariyoshi for assistance with experiments, and Rie Ikeyama and Yuka Mizutani for secretarial support.

\section{SUPPLEMENTARY MATERIAL}

The Supplementary Material for this article can be found online at: http://journal.frontiersin.org/article/10.3389/fphar. 2016.00206

\section{REFERENCES}

Ahir, B. K., Sanders, A. P., Rager, J. E., and Fry, R. C. (2013). Systems biology and birth defects prevention: blockade of the glucocorticoid receptor prevents arsenic-induced birth defects. Environ. Health Perspect. 121, 332-338. doi: 10.1289/ehp.1205659

Antherieu, S., Rogue, A., Fromenty, B., Guillouzo, A., and Robin, M. A. (2011). Induction of vesicular steatosis by amiodarone and tetracycline is associated with up-regulation of lipogenic genes in HepaRG cells. Hepatology 53, 18951905. doi: 10.1002/hep. 24290

Bai, Q., Garver, J. A., Hukriede, N. A., and Burton, E. A. (2007). Generation of a transgenic zebrafish model of Tauopathy using a novel promoter element derived from the zebrafish eno2 gene. Nucleic Acids Res. 35, 6501-6516. doi: $10.1093 / \mathrm{nar} / \mathrm{gkm} 608$

Barrett, T., Troup, D. B., Wilhite, S. E., Ledoux, P., Rudnev, D., Evangelista, C., et al. (2009). NCBI GEO: archive for high-throughput functional genomic data. Nucleic Acids Res. 37, D885-D890. doi: 10.1093/nar/gkn764

Barros, T. P., Alderton, W. K., Reynolds, H. M., Roach, A. G., and Berghmans, S. (2008). Zebrafish: an emerging technology for in vivo pharmacological assessment to identify potential safety liabilities in early drug discovery. $\mathrm{Br}$. J. Pharmacol. 154, 1400-1413. doi: 10.1038/bjp.2008.249

Bennett, M. K., Toth, J. I., and Osborne, T. F. (2004). Selective association of sterol regulatory element-binding protein isoforms with target promoters in vivo. J. Biol. Chem. 279, 37360-37367. doi: 10.1074/jbc.M40469 3200

Berger, J., Pujol, A., Aubourg, P., and Forss-Petter, S. (2010). Current and future pharmacological treatment strategies in X-linked adrenoleukodystrophy. Brain Pathol. 20, 845-856. doi: 10.1111/j.1750-3639.2010.00393.x

Besson, V. C., Chen, X. R., Plotkine, M., and Marchand-Verrecchia, C. (2005). Fenofibrate, a peroxisome proliferator-activated receptor alpha agonist, exerts neuroprotective effects in traumatic brain injury. Neurosci. Lett. 388, 7-12. doi: 10.1016/j.neulet.2005.06.019

Bindea, G., Mlecnik, B., Hackl, H., Charoentong, P., Tosolini, M., Kirilovsky, A., et al. (2009). ClueGO: a Cytoscape plug-in to decipher functionally grouped gene ontology and pathway annotation networks. Bioinformatics 25, 1091-1093. doi: 10.1093/bioinformatics/btp101

Buckley, C. E., Marguerie, A., Roach, A. G., Goldsmith, P., Fleming, A., Alderton, W. K., et al. (2010). Drug reprofiling using zebrafish identifies novel compounds with potential pro-myelination effects. Neuropharmacology 59, 149-159. doi: 10.1016/j.neuropharm.2010.04.014

Chew, L. J., and DeBoy, C. A. (2015). Pharmacological approaches to intervention in hypomyelinating and demyelinating white matter pathology. Neuropharmacology doi: 10.1016/j.neuropharm.2015.06.008 [Epub ahead of print].

Davis, A. P., Grondin, C. J., Lennon-Hopkins, K., Saraceni-Richards, C., Sciaky, D., King, B. L., et al. (2015). The Comparative Toxicogenomics Database's 10th year anniversary: update 2015. Nucleic Acids Res. 43, D914-D920. doi: 10.1093/nar/gku935
FIGURE S1 | Effects of fenofibrate and gemfibrozil on mbp promoter-driven fluorescence in zebrafish. $(A, B)$ Quantification of mCitrine fluorescence intensity within the area of Cerulean fluorescence. (A) Zebrafish were untreated $(n=9)$ or treated with fenofibrate ( $n=12$ for $100 \mathrm{nM}, n=7$ for $400 \mathrm{nM}$, $n=15$ for $700 \mathrm{nM})$. (B) Zebrafish were untreated $(n=17)$ or treated with gemfibrozil ( $n=11$ for $250 \mathrm{nM}, n=6$ for $500 \mathrm{nM}, n=14$ for $750 \mathrm{nM}$ ). ${ }^{* *} p<0.01,{ }^{* * *} p<0.001$ compared with control.

FIGURE S2 | Bright-field images of zebrafish treated with various drugs. Zebrafish were treated with the indicated drugs from $10 \mathrm{~h}$ post-fertilization to $5 \mathrm{dpf}$ and bright-field images were captured at $5 \mathrm{dpf}$.

FIGURE S3 | Quantitative PCR (qPCR) analysis of $h m g c r$, dhcr7, and $m b p$ in zebrafish treated with fenofibrate or gemfibrozil. qPCR analysis of $h m g \mathrm{cr}$, $d h c r 7$, and mbp mRNA levels in zebrafish treated with or without fenofibrate or gemfibrozil from $10 \mathrm{~h}$ post-fertilization to $5 \mathrm{dpf}$. Expression was normalized to ef1a mRNA levels. $n=6$ per group. If two groups do not share a letter, the difference between them is significant.

Deshmukh, V. A., Tardif, V., Lyssiotis, C. A., Green, C. C., Kerman, B., Kim, H. J., et al. (2013). A regenerative approach to the treatment of multiple sclerosis. Nature 502, 327-332. doi: 10.1038/nature12647

Esterberg, R., Coffin, A. B., Ou, H., Simon, J. A., Raible, D. W., and Rubel, E. W. (2013). Fish in a dish: drug discovery for hearing habilitation. Drug Discov. Today Dis. Models 10, e23-e29. doi: 10.1016/j.ddmod.2012.02.001

Eto, M., Sumi, H., Fujimura, H., Yoshikawa, H., and Sakoda, S. (2008). Pioglitazone promotes peripheral nerve remyelination after crush injury through CD36 upregulation. J. Peripher. Nerv. Syst. 13, 242-248. doi: 10.1111/j.15298027.2008.00183.x

Ferno, J., Skrede, S., Vik-Mo, A. O., Havik, B., and Steen, V. M. (2006). Druginduced activation of SREBP-controlled lipogenic gene expression in CNSrelated cell lines: marked differences between various antipsychotic drugs. $B M C$ Neurosci. 7:69. doi: 10.1186/1471-2202-7-69

Friedland, S. N., Leong, A., Filion, K. B., Genest, J., Lega, I. C., Mottillo, S., et al. (2012). The cardiovascular effects of peroxisome proliferator-activated receptor agonists. Am. J. Med. 125, 126-133. doi: 10.1016/j.amjmed.2011.08.025

Garbay, B., Boiron-Sargueil, F., Shy, M., Chbihi, T., Jiang, H., Kamholz, J., et al. (1998). Regulation of oleoyl-CoA synthesis in the peripheral nervous system: demonstration of a link with myelin synthesis. J. Neurochem. 71, 1719-1726. doi: 10.1046/j.1471-4159.1998.71041719.x

Gerstein, M. B., Kundaje, A., Hariharan, M., Landt, S. G., Yan, K. K., Cheng, C., et al. (2012). Architecture of the human regulatory network derived from ENCODE data. Nature 489, 91-100. doi: 10.1038/nature11245

Gilbert, M. E., Rovet, J., Chen, Z., and Koibuchi, N. (2012). Developmental thyroid hormone disruption: prevalence, environmental contaminants and neurodevelopmental consequences. Neurotoxicology 33, 842-852. doi: 10.1016/j.neuro.2011.11.005

Gunnarsson, L., Jauhiainen, A., Kristiansson, E., Nerman, O., and Larsson, D. G. (2008). Evolutionary conservation of human drug targets in organisms used for environmental risk assessments. Environ. Sci. Technol. 42, 5807-5813. doi: $10.1021 /$ es 8005173

Halder, S. K., Fink, M., Waterman, M. R., and Rozman, D. (2002). A cAMPresponsive element binding site is essential for sterol regulation of the human lanosterol 14alpha-demethylase gene (CYP51). Mol. Endocrinol. 16, 1853-1863. doi: 10.1210/me.2001-0262

Helenius, I. T., and Yeh, J. R. (2012). Small zebrafish in a big chemical pond. J. Cell. Biochem. 113, 2208-2216. doi: 10.1002/jcb.24120

Ikeda, Y., Yamamoto, J., Okamura, M., Fujino, T., Takahashi, S., Takeuchi, K., et al. (2001). Transcriptional regulation of the murine acetyl-CoA synthetase 1 gene through multiple clustered binding sites for sterol regulatory element-binding proteins and a single neighboring site for Sp1. J. Biol. Chem. 276, 34259-34269. doi: $10.1074 /$ jbc.M103848200

Jagannathan, N. R., Tandon, N., Raghunathan, P., and Kochupillai, N. (1998). Reversal of abnormalities of myelination by thyroxine therapy in congenital hypothyroidism: localized in vivo proton magnetic resonance spectroscopy (MRS) study. Brain Res. Dev. Brain Res. 109, 179-186. doi: 10.1016/S01653806(98)00081-9 
Jana, M., Mondal, S., Gonzalez, F. J., and Pahan, K. (2012). Gemfibrozil, a lipid-lowering drug, increases myelin genes in human oligodendrocytes via peroxisome proliferator-activated receptor-beta. J. Biol. Chem. 287, 3413434148. doi: 10.1074/jbc.M112.398552

Janky, R., Verfaillie, A., Imrichova, H., Van De Sande, B., Standaert, L., Christiaens, V., et al. (2014). iRegulon: from a gene list to a gene regulatory network using large motif and track collections. PLoS Comput. Biol. 10:e1003731. doi: 10.1371/journal.pcbi.1003731

Jung, S. H., Kim, S., Chung, A. Y., Kim, H. T., So, J. H., Ryu, J., et al. (2010). Visualization of myelination in GFP-transgenic zebrafish. Dev. Dyn. 239, 592597. doi: $10.1002 /$ dvdy.22166

Kamisuki, S., Mao, Q., Abu-Elheiga, L., Gu, Z., Kugimiya, A., Kwon, Y., et al. (2009). A small molecule that blocks fat synthesis by inhibiting the activation of SREBP. Chem. Biol. 16, 882-892. doi: 10.1016/j.chembiol.2009. 07.007

Kawakami, K. (2007). Tol2: a versatile gene transfer vector in vertebrates. Genome Biol. 8(Suppl. 1), S7. doi: 10.1186/gb-2007-8-1-r7

Kelsh, R. N., Brand, M., Jiang, Y. J., Heisenberg, C. P., Lin, S., Haffter, P., et al. (1996). Zebrafish pigmentation mutations and the processes of neural crest development. Development 123, 369-389.

Knight, B. L., Hebbachi, A., Hauton, D., Brown, A. M., Wiggins, D., Patel, D. D., et al. (2005). A role for PPARalpha in the control of SREBP activity and lipid synthesis in the liver. Biochem. J. 389, 413-421. doi: 10.1042/BJ20041896

Liao, Y., Smyth, G. K., and Shi, W. (2013). The Subread aligner: fast, accurate and scalable read mapping by seed-and-vote. Nucleic Acids Res. 41, e108. doi: 10.1093/nar/gkt214

Liao, Y., Smyth, G. K., and Shi, W. (2014). featureCounts: an efficient general purpose program for assigning sequence reads to genomic features. Bioinformatics 30, 923-930. doi: 10.1093/bioinformatics/btt656

Mandrekar-Colucci, S., Sauerbeck, A., Popovich, P. G., and Mctigue, D. M. (2013). PPAR agonists as therapeutics for CNS trauma and neurological diseases. ASN Neuro 5:e00129. doi: 10.1042/AN20130030

Martens, K., Ver Loren Van Themaat, E., Van Batenburg, M. F., Heinaniemi, M., Huyghe, S., Van Hummelen, P., et al. (2008). Coordinate induction of PPAR alpha and SREBP2 in multifunctional protein 2 deficient mice. Biochim. Biophys. Acta 1781, 694-702. doi: 10.1016/j.bbalip.2008.07.010

Mathews, E. S., Mawdsley, D. J., Walker, M., Hines, J. H., Pozzoli, M., and Appel, B. (2014). Mutation of 3-hydroxy-3-methylglutaryl CoA synthase I reveals requirements for isoprenoid and cholesterol synthesis in oligodendrocyte migration arrest, axon wrapping, and myelin gene expression. J. Neurosci. 34, 3402-3412. doi: 10.1523/JNEUROSCI.4587-13.2014

Mathias, J. R., Saxena, M. T., and Mumm, J. S. (2012). Advances in zebrafish chemical screening technologies. Future Med. Chem. 4, 1811-1822. doi: $10.4155 /$ fmc. 12.115

Ministry of the Environment of Japan (2013). Standards Relating to the Care and Management of Laboratory Animals and Relief of Pain, Notice No.88, April 28, 2006, Revised August 30, 2013. Tokyo: Ministry of the Environment of Japan.

Ministry of the Environment of Japan (2014). The Law for the Humane Treatment and Management of Animals, Law No. 105, October 1, 1973, Revised May 30, 2014. Tokyo: Ministry of the Environment of Japan.

Najm, F. J., Madhavan, M., Zaremba, A., Shick, E., Karl, R. T., Factor, D. C., et al. (2015). Drug-based modulation of endogenous stem cells promotes functional remyelination in vivo. Nature 522, 216-220. doi: 10.1038/nature14335

Nishimura, Y., Inoue, A., Sasagawa, S., Koiwa, J., Kawaguchi, K., Kawase, R., et al. (2016). Using zebrafish in systems toxicology for developmental toxicity testing. Congenit. Anom. (Kyoto) 56, 18-27. doi: 10.1111/cga.12142

Nishimura, Y., Murakami, S., Ashikawa, Y., Sasagawa, S., Umemoto, N., Shimada, Y., et al. (2015a). Zebrafish as a systems toxicology model for developmental neurotoxicity testing. Congenit. Anom. (Kyoto) 55, 1-16. doi: $10.1111 /$ cga.12079

Nishimura, Y., Sasagawa, S., Ariyoshi, M., Ichikawa, S., Shimada, Y., Kawaguchi, K., et al. (2015b). Systems pharmacology of adiposity reveals inhibition of EP300 as a common therapeutic mechanism of caloric restriction and resveratrol for obesity. Front. Pharmacol. 6:199. doi: 10.3389/fphar.2015.00199

Norrmen, C., Figlia, G., Lebrun-Julien, F., Pereira, J. A., Trotzmuller, M., Kofeler, H. C., et al. (2014). mTORC1 controls PNS myelination along the mTORC1RXRgamma-SREBP-lipid biosynthesis axis in Schwann cells. Cell Rep. 9, 646660. doi: 10.1016/j.celrep.2014.09.001
Ogata, M., Tsujita, M., Hossain, M. A., Akita, N., Gonzalez, F. J., Staels, B., et al. (2009). On the mechanism for PPAR agonists to enhance ABCA1 gene expression. Atherosclerosis 205, 413-419. doi: 10.1016/j.atherosclerosis.2009.01.008

Pertusa, M., Morenilla-Palao, C., Carteron, C., Viana, F., and Cabedo, H. (2007). Transcriptional control of cholesterol biosynthesis in Schwann cells by axonal neuregulin 1. J. Biol. Chem. 282, 28768-28778. doi: 10.1074/jbc.M701878200

Porcu, G., Serone, E., De Nardis, V., Di Giandomenico, D., Lucisano, G., Scardapane, M., et al. (2015). Clobetasol and halcinonide act as smoothened agonists to promote myelin gene expression and RxRgamma receptor activation. PLoS ONE 10:e0144550. doi: 10.1371/journal.pone.0144550

Prabhu, A. V., Sharpe, L. J., and Brown, A. J. (2014). The sterol-based transcriptional control of human 7-dehydrocholesterol reductase (DHCR7): evidence of a cooperative regulatory program in cholesterol synthesis. Biochim. Biophys. Acta 1842, 1431-1439. doi: 10.1016/j.bbalip.2014.07.006

Preston, M. A., and Macklin, W. B. (2015). Zebrafish as a model to investigate CNS myelination. Glia 63, 177-193. doi: 10.1002/glia.22755

Rampler, H., Weinhofer, I., Netik, A., Forss-Petter, S., Brown, P. J., Oplinger, J. A., et al. (2003). Evaluation of the therapeutic potential of PPARalpha agonists for X-linked adrenoleukodystrophy. Mol. Genet. Metab. 80, 398-407. doi: 10.1016/j.ymgme.2003.09.002

Rennekamp, A. J., and Peterson, R. T. (2015). 15 years of zebrafish chemical screening. Curr. Opin. Chem. Biol. 24, 58-70. doi: 10.1016/j.cbpa.2014.10.025

Rihel, J., and Schier, A. F. (2012). Behavioral screening for neuroactive drugs in zebrafish. Dev. Neurobiol. 72, 373-385. doi: 10.1002/dneu.20910

Rolland, B., Marche, K., Cottencin, O., and Bordet, R. (2012). The PPARalpha agonist fenofibrate reduces prepulse inhibition disruption in a neurodevelopmental model of schizophrenia. Schizophr. Res. Treat. 2012:839853. doi: $10.1155 / 2012 / 839853$

Roy, A., Jana, M., Corbett, G. T., Ramaswamy, S., Kordower, J. H., Gonzalez, F. J., et al. (2013). Regulation of cyclic AMP response element binding and hippocampal plasticity-related genes by peroxisome proliferator-activated receptor alpha. Cell Rep. 4, 724-737. doi: 10.1016/j.celrep.2013.07.028

Sasagawa, S., Nishimura, Y., Hayakawa, Y., Murakami, S., Ashikawa, Y., Yuge, M., et al. (2016a). E2F4 promotes neuronal regeneration and functional recovery after spinal cord injury in zebrafish. Front. Pharmacol. 7:119. doi: 10.3389/fphar.2016.00119

Sasagawa, S., Nishimura, Y., Kon, T., Yamanaka, Y., Murakami, S., Ashikawa, Y. et al. (2016b). DNA damage reesponse Is involved in the developmental toxicity of mebendazole in zebrafish retina. Front. Pharmacol. 7:57. doi: 10.3389/fphar.2016.00057

Science Council of Japan (2006). Guidelines for Proper Conduct of Animal Experiments. Available at: http://www.scj.go.jp/ja/info/kohyo/pdf/kohyo-20k16-2e.pdf

Shannon, P., Markiel, A., Ozier, O., Baliga, N. S., Wang, J. T., Ramage, D. et al. (2003). Cytoscape: a software environment for integrated models of biomolecular interaction networks. Genome Res. 13, 2498-2504. doi: $10.1101 /$ gr.1239303

Steiner, J., Martins-De-Souza, D., Schiltz, K., Sarnyai, Z., Westphal, S., Isermann, B., et al. (2014). Clozapine promotes glycolysis and myelin lipid synthesis in cultured oligodendrocytes. Front. Cell. Neurosci. 8:384. doi: 10.3389 /fncel.2014.00384

Sun, J., Nishiyama, T., Shimizu, K., and Kadota, K. (2013). TCC: an R package for comparing tag count data with robust normalization strategies. BMC Bioinformatics 14:219. doi: 10.1186/1471-2105-14-219

Tabor, D. E., Kim, J. B., Spiegelman, B. M., and Edwards, P. A. (1999). Identification of conserved cis-elements and transcription factors required for sterol-regulated transcription of stearoyl-CoA desaturase 1 and 2. J. Biol. Chem. 274, 20603-20610. doi: 10.1074/jbc.274.29.20603

Trapnell, C., Pachter, L., and Salzberg, S. L. (2009). TopHat: discovering splice junctions with RNA-Seq. Bioinformatics 25, 1105-1111. doi: 10.1093/bioinformatics/btp120

Vallett, S. M., Sanchez, H. B., Rosenfeld, J. M., and Osborne, T. F. (1996). A direct role for sterol regulatory element binding protein in activation of 3-hydroxy3-methylglutaryl coenzyme A reductase gene. J. Biol. Chem. 271, 12247-12253. doi: $10.1074 /$ jbc.271.21.12247

van der Meer, D. L., Degenhardt, T., Vaisanen, S., De Groot, P. J., Heinaniemi, M., De Vries, S. C., et al. (2010). Profiling of promoter occupancy by PPARalpha in 
human hepatoma cells via ChIP-chip analysis. Nucleic Acids Res. 38, 2839-2850. doi: 10.1093/nar/gkq012

Verheijen, M. H., Camargo, N., Verdier, V., Nadra, K., De Preux Charles, A. S., Medard, J. J., et al. (2009). SCAP is required for timely and proper myelin membrane synthesis. Proc. Natl. Acad. Sci. U.S.A. 106, 21383-21388. doi: 10.1073/pnas.0905633106

Westerfield, M. (2007). A Guide for the Laboratory Use of Zebrafish (Danio rerio). Eugene, OR: University of Oregon Press.

Yan, F., Wang, Q., Xu, C., Cao, M., Zhou, X., Wang, T., et al. (2014). Peroxisome proliferator-activated receptor alpha activation induces hepatic steatosis, suggesting an adverse effect. PLoS ONE 9:e99245. doi: 10.1371/journal.pone.0099245

Yang, Y., Gocke, A. R., Lovett-Racke, A., Drew, P. D., and Racke, M. K. (2008). PPAR alpha regulation of the immune response and autoimmune encephalomyelitis. PPAR Res 2008:546753. doi: 10.1155/2008/546753

Yang, Y., Tong, Y., Gong, M., Lu, Y., Wang, C., Zhou, M., et al. (2014). Activation of PPARbeta/delta protects pancreatic beta cells from palmitate-induced apoptosis by upregulating the expression of GLP-1 receptor. Cell. Signal. 26, 268-278. doi: 10.1016/j.cellsig.2013.11.019
Ye, J., and DeBose-Boyd, R. A. (2011). Regulation of cholesterol and fatty acid synthesis. Cold Spring Harb. Perspect. Biol. 3:a004754. doi: 10.1101/cshperspect.a004754

Conflict of Interest Statement: The authors declare that the research was conducted in the absence of any commercial or financial relationships that could be construed as a potential conflict of interest.

The reviewer MB and handling Editor declared their shared affiliation, and the handling Editor states that the process nevertheless met the standards of a fair and objective review.

Copyright (c) 2016 Ashikawa, Nishimura, Okabe, Sasagawa, Murakami, Yuge, Kawaguchi, Kawase and Tanaka. This is an open-access article distributed under the terms of the Creative Commons Attribution License (CC BY). The use, distribution or reproduction in other forums is permitted, provided the original author(s) or licensor are credited and that the original publication in this journal is cited, in accordance with accepted academic practice. No use, distribution or reproduction is permitted which does not comply with these terms. 